\title{
METRICAL FIXED POINT THEOREMS VIA LOCALLY FINITELY $T$-TRANSITIVE BINARY RELATIONS UNDER CERTAIN CONTROL FUNCTIONS
}

\author{
AFTAB ALAM, MOHAMMAD ARIF, AND MOHAMMAD IMDAD \\ Received 05 December, 2017
}

\begin{abstract}
In this paper, we extend relation-theoretic contraction principle due to Alam and Imdad to a nonlinear contraction using a relatively weaker class of continuous control functions employing a locally finitely $T$-transitive binary relation, which improves the corresponding fixed point theorems especially due to: Alam and Imdad (J. Fixed Point Theory Appl. 17 (2015) 693702), Agarwal et al. (Applicable Analysis, 87 (1) (2008) 109-116), Berzig and Karapinar (Fixed Point Theory Appl. 2013:205 (2013) 18 pp), Berzig et al. (Abstr. Appl. Anal. 2014:259768 (2014) 12 pp) and Turinici (The Sci. World J. 2014:169358 (2014) 10 pp).
\end{abstract}

2010 Mathematics Subject Classification: 47H10; 54H25

Keywords: locally finitely $T$-transitive binary relations, control functions, $\mathcal{R}$-connected sets

\section{INTRODUCTION}

The classical Banach contraction principle [6] is a pivotal result of metric fixed point theory. Several extensions of this core result are available in the existing literature of metric fixed point theory. Browder [9] extended Banach contraction principle to a class of nonlinear contractions which was later improved by Boyd and Wong [8], Mukherjea [16] and Jotić [11]. On the other hand, Ran and Reurings [19] and Nieto and Rodríguez-Lopez [17] extended Banach contraction principle to ordered metric spaces. In this continuation, Agarwal et al. [1] proved some order-theoretic fixed point results under nonlinear contractions employing comparison as well as continuous control functions, which was latter refined by O'Regan and Petruşel [18].

Recently, Alam and Imdad [2,3] obtained yet another generalization of classical Banach contraction principle using an amorphous (arbitrary) binary relation. In doing so, the authors introduced the relation-theoretic analogues of certain involved metrical notions such as: contraction, completeness, continuity etc. In fact, under the universal relation, all newly defined notions reduce to their corresponding natural analogues. 
The aim of this paper, is to extend relation-theoretic contraction principle due to Alam and Imdad [2] to a suitable class of nonlinear contractions. In order to ascertain the existence of fixed points for linear contraction of a self-mapping $T$, the underlying binary relation is required to be $T$-closed, whereas for such type nonlinear contraction, transitivity of underlying relation is additionally required. Notice that transitivity requirement is very restrictive. With a view to employ an optimal condition of transitivity, we visited several similar results involving various types of transitive binary relations. Although, all such results are independently proved by their respective authors, yet all such results are in the same mode, which is evident from the fact that our results of this paper improve as well as unify them all.

\section{PRELIMINARIES}

Recall that for a given nonempty set $X$, a subset $\mathcal{R}$ of $X^{2}$ is called a binary relation on $X$. For simplicity, we sometimes write $x \mathcal{R} y$ instead of $(x, y) \in \mathcal{R}$. Given subset $E \subseteq X$ and a binary relation $\mathcal{R}$ on $X$, the restriction of $\mathcal{R}$ to $E$, denoted by $\left.\mathcal{R}\right|_{E}$, is defined as $\left.\mathcal{R}\right|_{E}=\mathcal{R} \cap E^{2}$. Indeed, $\left.\mathcal{R}\right|_{E}$ is a relation on $E$ induced by $\mathcal{R}$.

Out of various classes of binary relations in practice, the following ones are relevant to our presentation:

A binary relation $\mathcal{R}$ defined on a nonempty set $X$ is called

- amorphous if it has no specific property at all,

- universal if $\mathcal{R}=X^{2}$,

- empty if $\mathcal{R}=\varnothing$,

- reflexive if $(x, x) \in \mathcal{R} \forall x \in X$,

- symmetric if whenever $(x, y) \in \mathcal{R}$ implies $(y, x) \in \mathcal{R}$,

- antisymmetric if whenever $(x, y) \in \mathcal{R}$ and $(y, x) \in \mathcal{R}$ imply $x=y$,

- transitive if whenever $(x, y) \in \mathcal{R}$ and $(y, z) \in \mathcal{R}$ imply $(x, z) \in \mathcal{R}$,

- complete if $(x, y) \in \mathcal{R}$ or $(y, x) \in \mathcal{R} \forall x, y \in X$,

- partial order if $\mathcal{R}$ is reflexive, antisymmetric and transitive.

Throughout this paper, $\mathcal{R}$ stands for a nonempty binary relation but for the sake of simplicity, we often write 'binary relation' instead of 'nonempty binary relation'. Also, $\mathbb{N}$ stands for the set of natural numbers, while $\mathbb{N}_{0}$ for the set of whole numbers (i.e., $\mathbb{N}_{0}:=\mathbb{N} \cup\{0\}$ ).

Definition 1 ([10,15,22]). Let $X$ be a nonempty set equipped with a partial order $\preceq$. A self-mapping $T$ defined on $X$ is called increasing (or isotone or orderpreserving) if for any $x, y \in X$,

$$
x \preceq y \Rightarrow T(x) \preceq T(y) .
$$

The following notion is formulated by using a suitable property with a view to relax the continuity requirement of the underlying mapping especially in the hypotheses of a fixed point theorem due to Nieto and Rodríguez-López [17]. 
Definition 2 ([5]). Let $(X, d)$ be a metric space equipped with a partial order $\preceq$. We say that $(X, d, \preceq)$ has $I C U$ (increasing-convergence-upper bound) property if every increasing convergent sequence in $X$ is bounded above by its limit (as an upper bound).

Recall that a function $\phi:[0, \infty) \rightarrow[0, \infty)$ is called control function if $\phi(t)<t$ for each $t>0$. The following order-thoertic fixed point theorem is a consequence of Theorem 2.3 due to Agarwal et al. [1] using a continuous control function.

Theorem 1. Let $(X, d)$ be a metric space equipped with a partial order $\preceq$ and $T$ a self-mapping on $X$. Suppose that the following conditions hold:

(a) $(X, d)$ is complete,

(b) $T$ is increasing,

(c) either $T$ is continuous or $(X, d, \preceq)$ has ICU property,

(d) there exists a continuous control function $\phi$ such that $d(T x, T y) \leq \phi(d(x, y)) \forall x, y \in X$ with $x \preceq y$.

Then $T$ has a fixed point.

In 2014, Berzig and Karapinar [14] introduced the idea of finitely transitive binary relation and proved some fixed point theorems in complete metric spaces endowed with a pair $\left(\mathcal{R}_{1}, \mathcal{R}_{2}\right)$ of finitely transitive binary relations under the class of $(\alpha \psi, \beta \varphi)$ contractive conditions.

Definition 3 ([7]). Given $N \in \mathbb{N}_{0}, N \geq 2$, a binary relation $\mathcal{R}$ defined on a nonempty set $X$ is called $N$-transitive if for any $x_{0}, x_{1}, \ldots, x_{N} \in X$,

$$
\left(x_{i-1}, x_{i}\right) \in \mathcal{R} \text { for each } i(1 \leq i \leq N) \Rightarrow\left(x_{0}, x_{N}\right) \in \mathcal{R} .
$$

Notice that, 2-transitivity coincides with transitivity. Following Turinici [26], $\mathcal{R}$ is called finitely transitive if it is $N$-transitive for some $N \geq 2$.

Definition 4 ([7]). Let $X$ be a nonempty set and $\mathcal{R}$ a binary relation on $X$. We say that a mapping $T: X \rightarrow X$ is an $\mathcal{R}$-preserving if for all $x, y \in X$,

$$
(x, y) \in \mathcal{R} \Rightarrow(T x, T y) \in \mathcal{R} .
$$

Definition 5 ( [7]). Let $(X, d)$ be a metric space and $\mathcal{R}_{1}$ and $\mathcal{R}_{2}$ two binary relations on $X$. We say that $(X, d)$ is $\left(\mathcal{R}_{1}, \mathcal{R}_{2}\right)$-regular if for every sequence $\left\{x_{n}\right\} \subseteq X$ such that $x_{n} \rightarrow x \in X$ as $n \rightarrow \infty$ and

$$
\left(x_{n}, x_{n+1}\right) \in \mathcal{R}_{1} \text { and }\left(x_{n}, x_{n+1}\right) \in \mathcal{R}_{2} \text { for all } n \in \mathbb{N},
$$

there exists a subsequence $\left\{x_{n_{k}}\right\}$ of $\left\{x_{n}\right\}$ such that

$$
\left(x_{n_{k}}, x\right) \in \mathcal{R}_{1} \text { and }\left(x_{n_{k}}, x\right) \in \mathcal{R}_{2} \text { for all } n \in \mathbb{N} \text {. }
$$

Definition 6 ( [7]). We say that a subset $D$ of $X$ is $\left(\mathcal{R}_{1}, \mathcal{R}_{2}\right)$-directed if for all $x, y \in D$, there exists $z \in X$ such that

$$
(x, z) \in \mathcal{R}_{1},(y, z) \in \mathcal{R}_{1},(x, z) \in \mathcal{R}_{2},(y, z) \in \mathcal{R}_{2} .
$$


Definition 7 ( [7]). We say that the pair of functions $(\psi, \varphi)$ is a pair of generalized altering distance (where $\psi, \varphi:[0, \infty) \rightarrow[0, \infty)$ ) if the following hypotheses hold:

$\left(a_{1}\right) \psi$ is continuous,

$\left(a_{2}\right) \psi$ is increasing,

(a) $\lim _{n \rightarrow \infty} \varphi\left(t_{n}\right)=0 \Rightarrow \lim _{n \rightarrow \infty} t_{n}=0$.

Definition 8 ([7]). Let $(X, d)$ be a metric space and $T: X \rightarrow X$ a given mapping. We say that $T$ is $(\alpha \psi, \beta \varphi)$-contractive mapping if there exists a pair of generalized altering distance $(\psi, \varphi)$ such that

$$
d(T x, T y) \leq \alpha(x, y) \psi(d(x, y))-\beta(x, y) \varphi(d(x, y)) \forall x, y \in X,
$$

where $\alpha, \beta: X^{2} \rightarrow[0, \infty)$.

Theorem 2 ([7]). Let $(X, d)$ be a complete metric space and $T: X \rightarrow X$ an $(\alpha \psi, \beta \varphi)$-contractive mapping. Let $\mathcal{R}_{1}$ and $\mathcal{R}_{2}$ be two binary relations on $X$ defined by $x, y \in X:(x, y) \in \mathcal{R}_{1} \Leftrightarrow \alpha(x, y) \leq 1$ and $(x, y) \in \mathcal{R}_{2} \Leftrightarrow \beta(x, y) \geq 1$ satisfying the following conditions:

(i) $\mathcal{R}_{1}$ and $\mathcal{R}_{2}$ are finitely transitive,

(ii) $T$ is $\mathcal{R}_{1}$-preserving and $\mathcal{R}_{2}$-preserving,

(iii) either $T$ is continuous or $(X, d)$ is $\left(\mathcal{R}_{1}, \mathcal{R}_{2}\right)$-regular,

(iv) there exists $x_{0} \in X$ such that $\left(x_{0}, T x_{0}\right) \in \mathcal{R}_{1}$ and $\left(x_{0}, T x_{0}\right) \in \mathcal{R}_{2}$.

Then $T$ has a fixed point. Moreover, if $X$ is $\left(\mathcal{R}_{1}, \mathcal{R}_{2}\right)$-directed, then fixed point remains unique.

In 2014, Theorem 2 is refined and extended by Berzig et al. [14] employing a relatively more general $(\alpha \psi, \beta \varphi)$-contractivity condition. Turinici [26] also extended Theorem 2 using 'finitely transitivity' in a local way besides generalizing the contractivity condition due to Meir-Keeler model.

Definition 9 ([26]). A binary relation $\mathcal{R}$ defined on a nonempty set $X$ is called locally finitely transitive if for each (effectively) denumerable subset $E$ of $X$, there exists $N=N(E) \geq 2$, such that $\left.\mathcal{R}\right|_{E}$ is $N$-transitive.

In a book chapter, Turinici [25] discussed some fixed point results on metric spaces endowed with a locally finitely transitive binary relation. For the sake of brevity, we skip to record the fixed point theorems of Turinici contained in $[25,26]$ here.

The following notion originated from $T$-transitive subset of $X^{2}$ is essentially due to Roldán-López-de-Hierro et al. [20]:

Definition 10 ( [3]). Let $X$ be a nonempty set and $T$ a self-mapping on $X$. A binary relation $\mathcal{R}$ defined on $X$ is called $T$-transitive if for any $x, y, z \in X$,

$$
(T x, T y),(T y, T z) \in \mathcal{R} \Rightarrow(T x, T z) \in \mathcal{R} .
$$


Henceforth, the notions of ' $T$-transitivity' and 'locally finitely transitivity' are not only weaker as compared to transitivity but they are also independent of each other. In order to make them compatible, we introduce the following notion of transitivity:

Definition 11. Let $X$ be a nonempty set and $T$ a self-mapping on $X$. A binary relation $\mathcal{R}$ defined on $X$ is called locally finitely $T$-transitive if for each (effectively) denumerable subset $E$ of $T(X)$, there exists $N=N(E) \geq 2$, such that $\left.\mathcal{R}\right|_{E}$ is $N$ transitive.

The following result establishes the superiority of the idea of 'locally finitely $T$ transitivity' over other variants of 'transitivity':

Proposition 1. Let $X$ be a nonempty set, $\mathcal{R}$ a binary relation on $X$ and $T$ a selfmapping on $X$. Then

(i) $\mathcal{R}$ is $T$-transitive $\left.\Leftrightarrow \mathcal{R}\right|_{T(X)}$ is transitive,

(ii ) $\mathcal{R}$ is locally finitely $T$-transitive $\left.\Leftrightarrow \mathcal{R}\right|_{T(X)}$ is locally finitely transitive,

(iii) $\mathcal{R}$ is transitive $\Rightarrow \mathcal{R}$ is finitely transitive $\Rightarrow \mathcal{R}$ is locally finitely transitive $\Rightarrow$ $\mathcal{R}$ is locally finitely $T$-transitive,

(iv) $\mathcal{R}$ is transitive $\Rightarrow \mathcal{R}$ is $T$-transitive $\Rightarrow \mathcal{R}$ is locally finitely $T$-transitive.

\section{RELEVANT NOTIONS AND AUXILIARY RESULTS}

In this section, for the sake of completeness, we summarize some relevant definitions and basic results for our subsequent discussion:

Definition 12 ([2]). Let $\mathcal{R}$ be a binary relation on a nonempty set $X$ and $x, y \in X$. We say that $x$ and $y$ are $\mathcal{R}$-comparative if either $(x, y) \in \mathcal{R}$ or $(y, x) \in \mathcal{R}$. We denote it by $[x, y] \in \mathcal{R}$.

Definition 13 ([13]). Let $X$ be a nonempty set and $\mathcal{R}$ a binary relation on $X$.

(1) The inverse or transpose or dual relation of $\mathcal{R}$, denoted by $\mathcal{R}^{-1}$, is defined by $\mathcal{R}^{-1}=\left\{(x, y) \in X^{2}:(y, x) \in \mathcal{R}\right\}$.

(2) The symmetric closure of $\mathcal{R}$, denoted by $\mathcal{R}^{s}$, is defined to be the set $\mathcal{R} \cup \mathcal{R}^{-1}$ (i.e., $\mathcal{R}^{s}:=\mathcal{R} \cup \mathcal{R}^{-1}$ ). Indeed, $\mathcal{R}^{s}$ is the smallest symmetric relation on $X$ containing $\mathcal{R}$.

Proposition 2 ([2]). For a binary relation $\mathcal{R}$ defined on a nonempty set $X$,

$$
(x, y) \in \mathcal{R}^{s} \Longleftrightarrow[x, y] \in \mathcal{R} .
$$

Definition 14 ([2]). Let $X$ be a nonempty set and $\mathcal{R}$ a binary relation on $X$. A sequence $\left\{x_{n}\right\} \subset X$ is called $\mathcal{R}$-preserving if

$$
\left(x_{n}, x_{n+1}\right) \in \mathcal{R} \forall n \in \mathbb{N}_{0} .
$$

Definition 15 ([2]). Let $X$ be a nonempty set and $T$ a self-mapping on $X$. A binary relation $\mathcal{R}$ defined on $X$ is called $T$-closed if for any $x, y \in X$,

$$
(x, y) \in \mathcal{R} \Rightarrow(T x, T y) \in \mathcal{R} \text {. }
$$


Proposition 3 ([3]). Let $X$ be a nonempty set equipped with a binary relation $\mathcal{R}$ and $T$ a self-mapping on $X$ such that $\mathcal{R}$ is $T$-closed, then $\mathcal{R}^{s}$ is also $T$-closed.

Proposition 4 ([4]). Let $X$ be a nonempty set, $\mathcal{R}$ a binary relation on $X$ and $T$ a self-mapping on $X$. If $\mathcal{R}$ is $T$-closed, then for all $n \in \mathbb{N}_{0}, \mathcal{R}$ is also $T^{n}$-closed, where $T^{n}$ denotes $n$th iterate of $T$.

Definition $16([3])$. Let $(X, d)$ be a metric space and $\mathcal{R}$ a binary relation on $X$. We say that $(X, d)$ is $\mathcal{R}$-complete if every $\mathcal{R}$-preserving Cauchy sequence in $X$ converges.

Clearly, every complete metric space is $\mathcal{R}$-complete with respect to a binary relation $\mathcal{R}$. Particularly, under the universal relation the notion of $\mathcal{R}$-completeness coincides with usual completeness.

Definition 17 ([3]). Let $(X, d)$ be a metric space and $\mathcal{R}$ a binary relation on $X$ with $x \in X$. A mapping $T: X \rightarrow X$ is called $\mathcal{R}$-continuous at $x$ if for any $\mathcal{R}$-preserving sequence $\left\{x_{n}\right\}$ such that $x_{n} \stackrel{d}{\longrightarrow} x$, we have $T\left(x_{n}\right) \stackrel{d}{\longrightarrow} T(x)$. Moreover, $T$ is called $\mathcal{R}$-continuous if it is $\mathcal{R}$-continuous at each point of $X$.

Clearly, every continuous mapping is $\mathcal{R}$-continuous, for any binary relation $\mathcal{R}$. Particularly, under the universal relation the notion of $\mathcal{R}$-continuity coincides with usual continuity.

The following notion is a generalization of $d$-self-closedness of a partial order relation $(\preceq)$ contained in Turinici [23,24]:

Definition 18 ([2]). Let $(X, d)$ be a metric space. A binary relation $\mathcal{R}$ defined on $X$ is called $d$-self-closed if for any $\mathcal{R}$-preserving sequence $\left\{x_{n}\right\}$ such that $x_{n} \stackrel{d}{\longrightarrow} x$, there exists a subsequence $\left\{x_{n_{k}}\right\}$ of $\left\{x_{n}\right\}$ with $\left[x_{n_{k}}, x\right] \in \mathcal{R} \quad \forall k \in \mathbb{N}_{0}$.

Definition 19 ([21]). Let $X$ be a nonempty set and $\mathcal{R}$ a binary relation on $X$. A subset $E$ of $X$ is called $\mathcal{R}$-directed if for each $x, y \in E$, there exists $z \in X$ such that $(x, z) \in \mathcal{R}$ and $(y, z) \in \mathcal{R}$.

Definition 20 ([12]). Let $X$ be a nonempty set and $\mathcal{R}$ a binary relation on $X$. For $x, y \in X$, a path of length $k$ (where $k$ is a natural number) in $\mathcal{R}$ from $x$ to $y$ is a finite sequence $\left\{z_{0}, z_{1}, z_{2}, \ldots, z_{k}\right\} \subset X$ satisfying the following:

(i) $z_{0}=x$ and $z_{k}=y$,

(ii) $\left(z_{i}, z_{i+1}\right) \in \mathcal{R}$ for each $i(0 \leq i \leq k-1)$.

Notice that a path of length $k$ involves $k+1$ elements of $X$, although they are not necessarily distinct.

Definition 21 ([3]). Let $X$ be a nonempty set and $\mathcal{R}$ a binary relation on $X$. A subset $E$ of $X$ is called $\mathcal{R}$-connected if for each pair $x, y \in E$, there exists a path in $\mathcal{R}$ from $x$ to $y$. 
Given a binary relation $\mathcal{R}$ and a self-mapping $T$ on a nonempty set $X$, we use the following notations:

(i) $F(T)=$ the set of all fixed points of $T$,

(ii) $X(T, \mathcal{R}):=\{x \in X:(x, T x) \in \mathcal{R}\}$.

The following result is a relation-theoretic version of Banach contraction principle:

Theorem 3 ([2,3]). Let $(X, d)$ be a metric space, $\mathcal{R}$ a binary relation on $X$ and $T$ a self-mapping on $X$. Suppose that the following conditions hold:

(a) $(X, d)$ is $\mathcal{R}$-complete,

(b) $\mathcal{R}$ is $T$-closed,

(c) either $T$ is $\mathcal{R}$-continuous or $\mathcal{R}$ is $d$-self-closed,

(d) $X(T, \mathcal{R})$ is nonempty,

(e) there exists $\alpha \in[0,1)$ such that $d(T x, T y) \leq \alpha d(x, y) \forall x, y \in X$ with $(x, y) \in \mathcal{R}$.

Then $T$ has a fixed point. Moreover, if $X$ is $\mathcal{R}^{s}$-connected, then $T$ has a unique fixed point.

Consider the following family of control functions:

$\Phi=\left\{\phi:[0, \infty) \rightarrow[0, \infty): \phi(t)<t\right.$ for each $t>0$ and $\limsup _{r \rightarrow t} \phi(r)<t$ for each $\left.t>0\right\}$.

Proposition 5. If $(X, d)$ is a metric space, $\mathcal{R}$ is a binary relation on $X, T$ is a selfmapping on $X$ and $\phi \in \Phi$, then the following contractivity conditions are equivalent:

(I) $d(T x, T y) \leq \phi(d(x, y)) \forall x, y \in X$ with $(x, y) \in \mathcal{R}$,

(II) $d(T x, T y) \leq \phi(d(x, y)) \forall x, y \in X$ with $[x, y] \in \mathcal{R}$.

We skip the proof of above proposition as it is similar to that of Proposition 2.3 in [2].

Proposition 6 ([5]). Let $\phi \in \Phi$. If $\left\{t_{n}\right\} \subset(0, \infty)$ is a sequence such that $t_{n+1} \leq$ $\phi\left(t_{n}\right) \forall n \in \mathbb{N}_{0}$, then $\lim _{n \rightarrow \infty} t_{n}=0$.

Finally, we record the following two known results, which are needed in the proof of our main results:

Lemma 1 ([14]). Let $(X, d)$ be a metric space and $\left\{x_{n}\right\}$ a sequence in $X$. If $\left\{x_{n}\right\}$ is not a Cauchy sequence, then there exist $\epsilon>0$ and two subsequences $\left\{x_{n_{k}}\right\}$ and $\left\{x_{m_{k}}\right\}$ of $\left\{x_{n}\right\}$ such that

(i) $k \leq m_{k}<n_{k} \forall k \in \mathbb{N}$,

(ii) $d\left(x_{m_{k}}, x_{n_{k}}\right) \geq \epsilon$,

(iii) $d\left(x_{m_{k}}, x_{p_{k}}\right)<\epsilon \forall p_{k} \in\left\{m_{k}+1, m_{k}+2, \ldots, n_{k}-2, n_{k}-1\right\}$.

In addition to this, if $\left\{x_{n}\right\}$ also verifies $\lim _{n \rightarrow \infty} d\left(x_{n}, x_{n+1}\right)=0$, then

$$
\lim _{k \rightarrow \infty} d\left(x_{m_{k}}, x_{n_{k}+p}\right)=\epsilon \forall p \in \mathbb{N}_{0} .
$$


Lemma 2 ([26]). Let $X$ be a nonempty set, $\mathcal{R}$ a binary relation on $X$ and $\left\{z_{n}\right\}$ is an $\mathcal{R}$-preserving sequence in $X$. If $\mathcal{R}$ is a $N$-transitive on $Z:=\left\{z_{n}: n \in \mathbb{N}_{0}\right\}$ for some natural number $N \geq 2$, then

$$
\left(z_{n}, z_{n+1+r(N-1)}\right) \in \mathcal{R} \forall n, r \in \mathbb{N}_{0} .
$$

\section{MAIN RESULTS}

Firstly, we prove a result on the existence of fixed points for the class $\Phi$ (described earlier) of nonlinear contractions employing a locally finitely $T$-transitive binary relation, which runs as follows:

Theorem 4. Let $(X, d)$ be a metric space equipped with a binary relation $\mathcal{R}$ and $T$ a self-mapping on $X$. Suppose that the following conditions hold:

(a) $(X, d)$ is $\mathcal{R}$-complete,

(b) $\mathcal{R}$ is $T$-closed and locally finitely $T$-transitive,

(c) either $T$ is $\mathcal{R}$-continuous or $\mathcal{R}$ is $d$-self-closed,

(d) $X(T, \mathcal{R})$ is nonempty,

(e) there exists $\phi \in \Phi$ such that $d(T x, T y) \leq \phi(d(x, y)) \forall x, y \in X$ with $(x, y) \in \mathcal{R}$.

Then $T$ has a fixed point.

Proof. As $X(T, \mathcal{R})$ is nonempty, one can choose $x_{0} \in X(T, \mathcal{R})$. Construct a sequence $\left\{x_{n}\right\}$ of Picard iteration based at the initial point $x_{0}$, i.e.,

$$
x_{n}=T^{n}\left(x_{0}\right) \forall n \in \mathbb{N}_{0} .
$$

As $\left(x_{0}, T x_{0}\right) \in \mathcal{R}$, using $T$-closedness of $\mathcal{R}$ and Proposition 4, we get

$$
\left(T^{n} x_{0}, T^{n+1} x_{0}\right) \in \mathcal{R}
$$

so that

$$
\left(x_{n}, x_{n+1}\right) \in \mathcal{R} \forall n \in \mathbb{N}_{0} .
$$

Therefore the sequence $\left\{x_{n}\right\}$ is $\mathcal{R}$-preserving. Now, if $d\left(x_{n_{0}+1}, x_{n_{0}}\right)=0$ for some $n_{0} \in \mathbb{N}_{0}$, then in view of (4.1), we have

$$
T\left(x_{n_{0}}\right)=x_{n_{0}}
$$

so that $x_{n_{0}}$ is a fixed point of $T$ and hence we are done.

On the other hand, if $d\left(x_{n+1}, x_{n}\right)>0 \forall n \in \mathbb{N}_{0}$, then applying the contractivity condition (e) to (4.2), we deduce, for all $n \in \mathbb{N}_{0}$ that

$$
d\left(x_{n+2}, x_{n+1}\right) \leq \phi\left(d\left(x_{n+1}, x_{n}\right)\right) .
$$

Using (4.3) and Proposition 6, we have

$$
\lim _{n \rightarrow \infty} d\left(x_{n}, x_{n+1}\right)=0 .
$$


Now, we show that $\left\{x_{n}\right\}$ is a Cauchy sequence. In fact, suppose that $\left\{x_{n}\right\}$ is not Cauchy. Therefore, owing to Lemma 1, there exist $\epsilon>0$ and two subsequences $\left\{x_{n_{k}}\right\}$ and $\left\{x_{m_{k}}\right\}$ of $\left\{x_{n}\right\}$ such that $k \leq m_{k}<n_{k}, d\left(x_{m_{k}}, x_{n_{k}}\right) \geq \epsilon$ and $d\left(x_{m_{k}}, x_{p_{k}}\right)<\epsilon$ where $p_{k} \in\left\{m_{k}+1, m_{k}+2, \ldots, n_{k}-2, n_{k}-1\right\}$. Further, in view of (4.4) and Lemma 1 , we infer

$$
\lim _{k \rightarrow \infty} d\left(x_{m_{k}}, x_{n_{k}+p}\right)=\epsilon \forall p \in \mathbb{N}_{0} .
$$

In view of (4.1), $\left\{x_{n}\right\} \subset T(X)$ and hence the range $E:=\left\{x_{n}: n \in \mathbb{N}_{0}\right\}$ (of the sequence $\left.\left\{x_{n}\right\}\right)$ is a denumerable subset of $T(X)$. Hence by locally finitely $T$ transitivity of $\mathcal{R}$, there exists a natural number $N=N(E) \geq 2$, such that $\left.\mathcal{R}\right|_{E}$ is $N$-transitive.

As $m_{k}<n_{k}$ and $N-1>0$, we have by the Division Rule

$$
\begin{gathered}
n_{k}-m_{k}=(N-1)\left(\mu_{k}-1\right)+\left(N-\eta_{k}\right) \\
\mu_{k}-1 \geq 0,0 \leq N-\eta_{k}<N-1 \\
\Longleftrightarrow\left\{\begin{array}{c}
n_{k}+\eta_{k}=m_{k}+1+(N-1) \mu_{k} \\
\mu_{k} \geq 1,1<\eta_{k} \leq N .
\end{array}\right.
\end{gathered}
$$

Here $\mu_{k}$ and $\eta_{k}$ are suitable natural numbers such that $\eta_{k}$ can assume any finite positive integral value in interval $(1, N]$. Hence, without loss of generality, we can choose subsequences $\left\{x_{n_{k}}\right\}$ and $\left\{x_{m_{k}}\right\}$ of $\left\{x_{n}\right\}$ (satisfying (4.5)) such that $\eta_{k}$ remains constant, say $\eta$, which is independent of $k$. Write

$$
m_{k}^{\prime}=n_{k}+\eta=m_{k}+1+(N-1) \mu_{k}
$$

where $\eta(1<\eta \leq N)$ is constant.

Owing to (4.5) and (4.6), we obtain

$$
\lim _{k \rightarrow \infty} d\left(x_{m_{k}}, x_{m_{k}^{\prime}}\right)=\lim _{k \rightarrow \infty} d\left(x_{m_{k}}, x_{n_{k}+\eta}\right)=\epsilon .
$$

Using triangular inequality, we have

$$
\begin{aligned}
d\left(x_{m_{k}+1}, x_{m_{k}^{\prime}+1}\right) & \leq d\left(x_{m_{k}+1}, x_{m_{k}}\right)+d\left(x_{m_{k}}, x_{m_{k}^{\prime}}\right)+d\left(x_{m_{k}^{\prime}}, x_{m_{k}^{\prime}+1}\right) \text { and } \\
d\left(x_{m_{k}}, x_{m_{k}^{\prime}}\right) & \leq d\left(x_{m_{k}}, x_{m_{k}+1}\right)+d\left(x_{m_{k+1}}, x_{m_{k}^{\prime}+1}\right)+d\left(x_{m_{k}^{\prime}+1}, x_{m_{k}^{\prime}}\right),
\end{aligned}
$$

therefore, we have

$$
\begin{aligned}
d\left(x_{m_{k}}, x_{m_{k}^{\prime}}\right)-d\left(x_{m_{k}}, x_{m_{k}+1}\right)-d\left(x_{m_{k}^{\prime}+1}, x_{m_{k}^{\prime}}\right) \leq d\left(x_{m_{k}+1}, x_{m_{k}^{\prime}+1}\right) \\
\leq d\left(x_{m_{k}+1}, x_{m_{k}}\right)+d\left(x_{m_{k}}, x_{m_{k}^{\prime}}\right)+d\left(x_{m_{k}^{\prime}}, x_{m_{k}^{\prime}+1}\right)
\end{aligned}
$$

which on letting $k \rightarrow \infty$ and using (4.4) and (4.7), gives rise

$$
\lim _{k \rightarrow \infty} d\left(x_{m_{k}+1}, x_{m_{k}^{\prime}+1}\right)=\epsilon .
$$


In view (4.6) and Lemma 2, we have $\left(x_{m_{k}}, x_{m_{k}^{\prime}}\right) \in \mathcal{R}$. Denote $\delta_{k}=d\left(x_{m_{k}}, x_{m_{k}^{\prime}}\right)$. Now, owing to (4.1) and assumption (e), we have

$$
\begin{aligned}
d\left(x_{m_{k}+1}, x_{m_{k}^{\prime}+1}\right) & =d\left(T x_{m_{k}}, T x_{m_{k}^{\prime}}\right) \\
& \leq \phi\left(d\left(x_{m_{k}}, x_{m_{k}^{\prime}}\right)\right) \\
& =\phi\left(\delta_{k}\right)
\end{aligned}
$$

so that

$$
d\left(x_{m_{k}+1}, x_{m_{k}^{\prime}+1}\right) \leq \phi\left(\delta_{k}\right) .
$$

Utilizing $\delta_{k} \rightarrow \epsilon$ as $k \longrightarrow \infty$ (in view of (4.7)) and the definition of $\Phi$, we have

$$
\limsup _{k \rightarrow \infty} \phi\left(\delta_{k}\right)=\limsup _{t \rightarrow \epsilon} \phi(t)<\epsilon .
$$

On taking limit superior as $k \rightarrow \infty$ in (4.9) besides using (4.8) and (4.10), we obtain

$$
\epsilon=\limsup _{k \rightarrow \infty} d\left(x_{m_{k}+1}, x_{m_{k}^{\prime}+1}\right) \leq \limsup _{k \rightarrow \infty} \phi\left(\delta_{k}\right)<\epsilon,
$$

which is a contradiction so that the sequence $\left\{x_{n}\right\}$ is Cauchy. Hence, $\left\{x_{n}\right\}$ is an $\mathcal{R}$-preserving Cauchy sequence. By $\mathcal{R}$-completeness of $(X, d), \exists x \in X$ such that $x_{n} \stackrel{d}{\longrightarrow} x$.

Finally, we claim that $x$ is a fixed point of $T$. To substantiate this, suppose that $T$ is $\mathcal{R}$-continuous. As $\left\{x_{n}\right\}$ is $\mathcal{R}$-preserving with $x_{n} \stackrel{d}{\longrightarrow} x, \mathcal{R}$-continuity of $T$ implies that $x_{n+1}=T\left(x_{n}\right) \stackrel{d}{\longrightarrow} T(x)$. Using the uniqueness of limit, we obtain $T(x)=x$, i.e., $x$ is a fixed point of $T$.

Alternately, assume that $\mathcal{R}$ is $d$-self-closed. As $\left\{x_{n}\right\}$ is $\mathcal{R}$-preserving such that $x_{n} \stackrel{d}{\longrightarrow} x$, the $d$-self-closedness of $\mathcal{R}$ guarantees the existence of a subsequence $\left\{x_{n_{k}}\right\}$ of $\left\{x_{n}\right\}$ with $\left[x_{n_{k}}, x\right] \in \mathcal{R} \quad\left(\forall k \in \mathbb{N}_{0}\right)$. On using assumption (e), Proposition 5 and $\left[x_{n_{k}}, x\right] \in \mathcal{R}$, we have

$$
d\left(x_{n_{k}+1}, T x\right)=d\left(T x_{n_{k}}, T x\right) \leq \phi\left(d\left(x_{n_{k}}, x\right)\right) \quad \forall k \in \mathbb{N}_{0} .
$$

We assert that

$$
d\left(x_{n_{k}+1}, T x\right) \leq d\left(x_{n_{k}}, x\right) \quad \forall k \in \mathbb{N} .
$$

On account of two different possibilities occurring here, we consider a partition of $\mathbb{N}$ i.e., $\mathbb{N}^{0} \cup \mathbb{N}^{+}=\mathbb{N}$ and $\mathbb{N}^{0} \cap \mathbb{N}^{+}=\varnothing$ verifying that

(i) $d\left(x_{n_{k}}, x\right)=0 \quad \forall k \in \mathbb{N}^{0}$,

(ii) $d\left(x_{n_{k}}, x\right)>0 \quad \forall k \in \mathbb{N}^{+}$.

In case $(i)$, we have $d\left(T x_{n_{k}}, T x\right)=0 \leq d\left(x_{n_{k}}, x\right) \forall k \in \mathbb{N}^{0}$. In case (ii), and definition of $\Phi$, we have $d\left(x_{n_{k}+1}, T x\right) \leq \phi\left(d\left(x_{n_{k}}, x\right)\right)<d\left(x_{n_{k}}, x\right)$ for all $k \in \mathbb{N}^{+}$. Hence in both the cases, we get $d\left(x_{n_{k}+1}, T x\right) \leq d\left(x_{n_{k}}, x\right) \forall k \in \mathbb{N}$, which by using the fact that $x_{n_{k}} \stackrel{d}{\longrightarrow} x$ as $k \rightarrow \infty$, yields that $x_{n_{k}+1} \stackrel{d}{\longrightarrow} T(x)$. Again, owing to 
the uniqueness of limit, we obtain $T(x)=x$ so that $x$ is a fixed point of $T$. This completes the proof.

Remark 1 . Clearly, $\phi \in \Phi$ utilized in Theorem 4 can be alternatively replaced by the following ones:

(i) $\phi$ is a continuous control function,

(ii) $\phi$ is an upper semi continuous control function,

(iii) $\phi$ is a control function satisfying $\lim _{r \rightarrow t} \phi(r)<t$ for each $t>0$.

In view of above remark, that Theorem 4 extends Theorem 1 (by taking $\mathcal{R}=\preceq$, partial order).

In view of Proposition 1, we obtain the following consequence of Theorem 4.

Corollary 1. Theorem 4 remains true if locally finitely $T$-transitivity of $\mathcal{R}$ (utilized in assumption $(b))$ is replaced by any one of the following conditions (besides retaining rest of the hypotheses):

(i) $\mathcal{R}$ is transitive,

(ii) $\mathcal{R}$ is $T$-transitive,

(iii) $\mathcal{R}$ is finitely transitive,

(iv) $\mathcal{R}$ is locally finitely transitive.

Now, we establish a uniqueness result corresponding to Theorem 4.

Theorem 5. In addition to the hypotheses of Theorem 4, suppose that the following condition holds:

(u) : $T(X)$ is $\mathcal{R}^{s}$-connected.

Then $T$ has a unique fixed point.

Proof. In view of Theorem 4, $F(T) \neq \varnothing$. Take $x, y \in F(T)$, then for all $n \in \mathbb{N}_{0}$, we have

$$
T^{n}(x)=x \text { and } T^{n}(y)=y .
$$

By assumption $(u)$, there exists a path (say $\left\{z_{0}, z_{1}, z_{2}, \ldots, z_{k}\right\}$ ) of some finite length $k$ in $\mathcal{R}^{s}$ from $x$ to $y$ so that

$$
z_{0}=x, z_{k}=y \text { and }\left[z_{i}, z_{i+1}\right] \in \mathcal{R} \text { for each } i(0 \leq i \leq k-1) .
$$

As $\mathcal{R}$ is $T$-closed, using Propositions 3 and 4 , we have

$$
\left[T^{n} z_{i}, T^{n} z_{i+1}\right] \in \mathcal{R} \text { for each } i(0 \leq i \leq k-1) \text { and for each } n \in \mathbb{N}_{0} .
$$

Now, for each $n \in \mathbb{N}_{0}$ and for each $i(0 \leq i \leq k-1)$, write $t_{n}^{i}:=d\left(T^{n} z_{i}, T^{n} z_{i+1}\right)$. We assert that

$$
\lim _{n \rightarrow \infty} t_{n}^{i}=0
$$


With $i$ fixed, we distinguish two cases. Firstly, suppose that $t_{n_{0}}^{i}=d\left(T^{n_{0}} z_{i}\right.$, $\left.T^{n_{0}} z_{i+1}\right)=0$ for some $n_{0} \in \mathbb{N}_{0}$, i.e., $T^{n_{0}}\left(z_{i}\right)=T^{n_{0}}\left(z_{i+1}\right)$, which implies that $T^{n_{0}+1}\left(z_{i}\right)=T^{n_{0}+1}\left(z_{i+1}\right)$. Consequently, we get $t_{n_{0}+1}^{i}=d\left(T^{n_{0}+1} z_{i}, T^{n_{0}+1} z_{i+1}\right)=$

0 . Thus by induction on $n$, we get $t_{n}^{i}=0 \forall n \geq n_{0}$, so that $\lim _{n \rightarrow \infty} t_{n}^{i}=0$. Secondly, suppose that $t_{n}^{i}>0 \forall n \in \mathbb{N}_{0}$. Then on using (4.13), assumption (e) and Proposition 5 , we have

$$
\begin{aligned}
t_{n+1}^{i} & =d\left(T^{n+1} z_{i}, T^{n+1} z_{i+1}\right) \\
& \leq \phi\left(d\left(T^{n} z_{i}, T^{n} z_{i+1}\right)\right) \\
& =\phi\left(t_{n}^{i}\right)
\end{aligned}
$$

so that

$$
t_{n+1}^{i} \leq \phi\left(t_{n}^{i}\right)
$$

Using (4.15) and Proposition 6, we have

$$
\lim _{n \rightarrow \infty} t_{n}^{i}=0, \text { for each } i(0 \leq i \leq k-1) .
$$

Hence in both the cases, (4.14) is proved. Making use of (4.11), (4.12), (4.14) and the triangular inequality, we have

$$
d(x, y)=d\left(T^{n} z_{0}, T^{n} z_{k}\right) \leq t_{n}^{0}+t_{n}^{1}+\cdots+t_{n}^{k-1} \rightarrow 0 \text { as } n \rightarrow \infty
$$

so that $x=y$. Hence $T$ has a unique fixed point.

Corollary 2. Theorem 5 remains true if we replace condition $(u)$ by one of the following conditions (besides retaining rest of the hypotheses):

$\left.\left(u^{\prime}\right) \mathcal{R}\right|_{T(X)}$ is complete,

(u') $T(X)$ is $\mathcal{R}^{s}$-directed.

A proof of above corollary can be outlined on the lines of the proof of Corollary 3.4 contained in [4].

The following example is adopted to exhibit and substantiate the utility of Theorems 4 and 5 over corresponding earlier known results.

Example 1. Consider $X=[0, \infty)$ equipped with usual metric $d$. Define a mapping $T: X \rightarrow X$ by $T(x)=\frac{x}{x+1} \forall x \in X$. Let $\mathcal{R}:=\left\{(x, y) \in X^{2}: x-y>0\right\}$, then $\mathcal{R}$ is locally finitely $T$-transitive binary relation on $X$. Clearly $X$ is $\mathcal{R}$-complete and $\mathcal{R}$ is $T$-closed. Now, define a control function $\phi$ by $\phi(t)=\frac{t}{t+1} \forall t \in[0, \infty)$, clearly $\phi \in \Phi$. Now, for all $(x, y) \in \mathcal{R}$, we have

$$
\begin{aligned}
d(T x, T y)=\left|\frac{x}{x+1}-\frac{y}{y+1}\right| & =\left|\frac{x-y}{1+x+y+x y}\right| \\
& \leq \frac{x-y}{1+(x-y)}=\frac{d(x, y)}{1+d(x, y)}=\phi(d(x, y)) .
\end{aligned}
$$


Hence $T$ and $\phi$ satisfy the assumption (e) of Theorem 5. It is easy to see that, rest of the conditions of Theorem 5 are also satisfied and $T$ has a unique fixed point (namely: $x=0$ ). As the relation $\mathcal{R}$ is not partial order and also $T$ is not a linear contraction, Example 1 can not be covered by Theorems 1 and 3, which demonstrate the utility of our newly proved results.

Now, we deduce some special cases, which are well known fixed point theorems of the existing literature.

(1) Under the universal relation (i.e., $\mathcal{R}=X^{2}$ ), Theorem 5 deduces to the classical fixed point theorem under nonlinear contraction.

(2) Taking $\phi(t)=\alpha t$ (where $\alpha \in[0,1)$ ), we obtain Theorem 3. In this case, the requirement of locally finitely $T$-transitivity on a binary relation is not necessary.

(3) Putting $\mathcal{R}=\mathcal{R}_{1} \cap \mathcal{R}_{2}$ (where $\mathcal{R}_{1}$ and $\mathcal{R}_{2}$ are finitely transitive) in Theorem 4, we obtain an analogue of Theorem 2 (under the class $\Phi$ ). Notice that $T$-closedness of $\mathcal{R}$ is equivalent to $\mathcal{R}$-preserving property of $T$ and $d$-selfclosedness of $\mathcal{R}$ is slightly weaker than $\left(\mathcal{R}_{1}, \mathcal{R}_{2}\right)$-regularity of $(X, d)$.

(4) Setting $\mathcal{R}$ to be locally finitely transitive binary relation in Theorem 4, we obtain an analogue of a fixed point theorem of Turinici [26] for the nonlinear class $\Phi$.

In an attempt to extend Theorem 3 from linear contractions to a nonlinear contractions (under the class $\Phi$ ), we additionally do require locally finitely $T$-transitivity of $\mathcal{R}$ (optimal condition), which substantiates the utility of the present extension. For possible problems, readers may attempt to prove such results for other type of nonlinear contractions.

\section{ACKNOWLEDGMENTS}

All the authors are gratefull to an anonymous learned referee for his/her critical readings and pertinent comments besides pointing out a fatal error. The second author is thankful to University Grant Commission, New Delhi, Government of India for the financial support in the form of MANF (Moulana Azad National Fellowship).

\section{REFERENCES}

[1] R. P. Agarwal, M. A. El-Gebeily, and D. O'Regan, "Generalized contractions in partially ordered metric spaces," Applicable Analysis., vol. 87, no. 1, pp. 109-116, 2008.

[2] A. Alam and M. Imdad, "Relation-theoretic contraction principle." J. Fixed Point Theory Appl., vol. 17 , no. 4, pp. 693-702, 2015.

[3] A. Alam and M. Imdad, "Relation-theoretic metrical coincidence theorems." Filomat., vol. 31, no. 14 , pp. 4421-4439, 2017.

[4] A. Alam and M. Imdad, "Nonlinear contractions in metric spaces under locally T-transitive binary relations." Fixed Point Theory, vol. 19, no. 1, pp. 13-24, 2018. 
[5] A. Alam, A. R. Khan, and M. Imdad, "Some coincidence theorems for generalized nonlinear contractions in ordered metric spaces with applications." Fixed Point Theory Appl., vol. 2014, 2014, art.no. 216, 30pp.

[6] S. Banach, "Sur les operations dans les ensembles abstraits et leur application aux equations intgerales." Fund. Math., vol. 3, no. 1, pp. 133-181, 1922.

[7] M. Berzig and E. Karapinar, "Fixed point results for $(\alpha \psi, \beta \varphi)$ - contractive mappings for a generalized altering distance." Fixed Point Theory Appl., vol. 2013, 2013, art. no 205, 18pp.

[8] D. W. Boyd and J. S. W. Wong, "On nonlinear contractions." Proc. Amer. Math. Soc., vol. 30, no. $27,1969,25 \mathrm{pp}$.

[9] F. E. Browder, "On the convergence of successive approximations for nonlinear functional equations." Proc. K. Ned. Akad. Wet. Ser. A, Indag. Math., vol. 71, pp. 27-35, 1968.

[10] R. DeMarr, "Common fixed points for isotone mappings." Colloq. Math., vol. 13, no. 1, pp. 45-48, 1964.

[11] N. Jotić, "Some fixed point theorems in metric spaces." Indian J. Pure Appl. Math., vol. 26, pp. 947-952, 1995.

[12] B. Kolman, R. C. Busby, and S. Ross, "Discrete mathematical structures.” Third Edition, PHI Pvt. Ltd., New Delhi., 2000.

[13] S. Lipschutz, Schaum's outlines of theory and problems of set theory and related topics. New York: McGraw-Hill, New York, 1964.

[14] M. Berzig, E. Karapinar, and A. Roldán, "Discussion on generalized- $(\alpha \psi, \beta \varphi)$-contractive mappings via generalized altering distance function and related fixed point theorems." Abstr. Appl. Anal., vol. 2014, 2014, art. no.259768, 12pp.

[15] E. J. McShane, "Order-preserving maps and integration processes." Ann. Math. St. 31, Princeton University Press, Princeton., vol. 31, 1953.

[16] A. Mukherjea, "Contractions and completely continuous mappings." Nonlinear Analysis: Theory, Methods \& Applications., vol. 1, no. 3, pp. 235-247, 1977.

[17] J. J. Nieto and R. Rodríguez-López, "Contractive mapping theorems in partially ordered sets and applications to ordinary differential equations." Order, vol. 22, no. 3, pp. 223-239, 2005.

[18] D. O'Regan and A. Petruşel, "Fixed point theorems for generalized contractions in ordered metric spaces." J. Math. Anal. Appl., vol. 341, no. 2, pp. 1241-1252, 2008.

[19] A. Ran and M. Reurings, "A fixed point theorem in partially ordered sets and some applications to matrix equations." Proc. Am. Math. Soc., vol. 132, no. 5, pp. 1435-1443, 2004.

[20] A. F. Roldán-López-de-Hierro, E. Karapinar, and M. de-la-Sen, " Coincidence point theorems in quasi-metric spaces without assuming the mixed monotone property and consequences in Gmetric spaces." Fixed Point Theory Appl., vol. 2014, 2014, art. no. 184, 29pp.

[21] B. Samet and M. Turinici, "Fixed point theorems on a metric space endowed with an arbitrary binary relation and applications." Commun. Math. Anal., vol. 13, no. 2, pp. 82-97, 2012.

[22] A. Tarski, " A lattice-theoretical fixpoint theorem and its applications." Pacific J. Math., vol. 5, no. 2, pp. 285-309, 1955.

[23] M. Turinici, "Product fixed points in ordered metric spaces." arXiv:1110.3079v1., 2011.

[24] M. Turinici, "Linear contractions in product ordered metric spaces." Ann Univ Ferrara., vol. 59, no. 1, pp. 187-198, 2013.

[25] M. Turinici, " Contractive operators in relational metric spaces." Handbook of Functional Equations, Springer Optimization and its Applications, Springer, vol. 95, pp. 419-458, 2014.

[26] M. Turinici, "Contractive maps in locally transitive relational metric spaces." The Sci. World J., vol. 2014, 2014, art. no. 169358, 10pp. 
Authors' addresses

Aftab Alam

Department of Mathematics, Aligarh Muslim University, Aligarh-202002, India

E-mail address: aafu.amu@gmail.com

Mohammad Arif

Department of Mathematics, Aligarh Muslim University, Aligarh-202002, India

E-mail address: mohdarif154c@gmail

Mohammad Imdad

Department of Mathematics, Aligarh Muslim University, Aligarh-202002, India

E-mail address: mhimdad@gmail.com 